\title{
Thermal infrared observations of near-Earth asteroid 2002 NY40^
}

\author{
T. G. Müller ${ }^{1}$, M. F. Sterzik ${ }^{2}$, O. Schütz ${ }^{3}$, P. Pravec ${ }^{4}$, and R. Siebenmorgen ${ }^{5}$ \\ 1 Max-Planck-Institut für extraterrestrische Physik, Giessenbachstraße, 85748 Garching, Germany \\ e-mail: tmueller@mpe.mpg.de \\ 2 European Southern Observatory, Casilla 19001, Santiago 19, Chile \\ e-mail: msterzik@eso.org \\ 3 Max-Planck-Institut für Astronomie, Königstuhl 17, 69117 Heidelberg, Germany \\ e-mail: schuetz@mpia-hd.mpg.de \\ 4 Astronomical Institute, Academy of Sciences of the Czech Republic, Ondrejov, 25165, Czech Republic \\ e-mail: ppravec@asu.cas.cz \\ 5 European Southern Observatory, Karl-Schwarzschildstr. 2, 85748 Garching, Germany \\ e-mail: rsiebenm@eso.org
}

Received 8 April 2004 / Accepted 3 June 2004

\begin{abstract}
We obtained N-band observations of the Apollo asteroid 2002 NY40 during its close Earth fly-by in August 2002 with TIMMI2 at the ESO $3.6 \mathrm{~m}$ telescope. The photometric measurement allowed us to derive a radiometric diameter of $0.28 \pm$ $0.03 \mathrm{~km}$ and an albedo of $0.34 \pm 0.06$ through the near-Earth asteroid thermal model (NEATM) and a thermophysical model (TPM). The values are in agreement with results from radar data, visual and near-IR observations. In this first comparison between these two model approaches we found that the empirical NEATM beaming parameter $\eta=1.0$ corresponds to a thermal inertia values of about $100 \mathrm{~J} \mathrm{~m}^{-2} \mathrm{~s}^{-0.5} \mathrm{~K}^{-1}$ for a typical range of surface roughness, assuming an equator-on viewing angle. Our TPM analysis indicated that the surface of 2002 NY40 consists of rocky material with a thin or no dust regolith. The asteroid very likely has a prograde sense of rotation with a cold terminator at the time of our observations. Although both model approaches can fit the thermal spectra taken at phase angles of $22^{\circ}$ and $59^{\circ}$, we did not find a consistent model solution that describes all pieces of photometric and spectroscopic data. In addition to the 2002 NY40 analysis, we discuss the possibilities to distinguish between different models with only very few photometric and/or spectroscopic measurements spread over a range of phase angles.
\end{abstract}

Key words. minor planets, asteroids - radiation mechanisms: thermal - infrared: solar system

\section{Introduction}

The Apollo asteroid 2002 NY40 was discovered by LINEAR ${ }^{1}$ (MPEC 2002-O172). It passed the Earth at $0.0035 \mathrm{AU}$, about 1.3 times the distance to the Moon, on August 18th, 2002. This event was closely followed by many amateur and professional observers at a large range of wavelengths from visible to radar.

Pravec et al. (2004) found from 21 different observing sessions a main lightcurve period of $P_{1}=19.98 \pm 0.01 \mathrm{~h}$ with indications for a non-principal axis rotation with a nonunique but likely second period of $P_{2}=18.43 \pm 0.01 \mathrm{~h}$. The lightcurve shows sharp bends, indicating the presence of large non-convex shape features. Its mean absolute magnitude is $H=19.23 \pm 0.2 \mathrm{mag}$, with variations between $18.9 \mathrm{mag}$ for the lightcurve maximum and $19.8 \mathrm{mag}$ at the secondary minimum (see Bowell et al. 1989 for a definition of the H-G system).

\footnotetext{
* Based on observations collected at the European Southern Observatory, Chile; ESO, No. 69.C-0152.

${ }^{1}$ Lincoln Near Earth Asteroid Research project.

${ }^{2}$ http://cfa-www.harvard.edu/mpec/KQ2/K02017.html
}

Howell et al. (2003) presented radar images which show that 2002 NY40 looks like two spheroidal units joined together, but high resolution optical imaging showed no obvious evidence for a binary structure $\left(\mathrm{H}\right.$. Mathis $\left.{ }^{3}\right)$.

0.3 to $4.0 \mu \mathrm{m}$ spectroscopy revealed absorption bands at 1 and $2 \mu \mathrm{m}$ due to olivine and pyroxene (Rivkin et al. 2003). The striking similarity with LL6 chondrites, a subset of ordinary chondrites, indicates that 2002 NY40 could be a progenitor of LL6 meteorites. Based on the visible spectrum, Rivkin et al. (2003) classified 2002 NY40 as Q-class asteroid, which is connected to a moderately high albedo of $20-30 \%$ (Clark et al. 2002).

First size estimates were in the order of $700 \mathrm{~m}$ to $800 \mathrm{~m}$ diameter ${ }^{3,4}$, based on magnitude estimates from early astrometric measurements. Later on, adaptive optics systems lowered this number to an upper limit of $400 \mathrm{~m}$ at the time of

\footnotetext{
${ }^{3}$ http://www. noao.edu/outreach/press/pr02/pr0207.html

${ }^{4}$ http://science.nasa.gov/headlines/y2002/

30jul_ny40.html
} 
the observation ${ }^{5}$. Radar data lead to a size estimate of about $250 \times 420 \mathrm{~m}$ (E. Howell, personal communication) consistent with radiometric results (Rivkin et al. 2003) from $2.5 \mu \mathrm{m}$ observations. They also gave a possible albedo range of $0.15-0.25$ with a slight preference for the higher value.

The radiometric method of diameter and albedo determination from thermal radiation measurements goes back to the early 1970s (references are given in e.g., Morrison \& Lebofsky 1979). A widespread version of this technique, the Standard Thermal Model (STM), was published by Lebofsky et al. (1986) and discussed by e.g., Lebofsky \& Spencer (1989). The STM uses an empirical beaming parameter $\eta$, which was introduced to adjust the sub-solar surface temperature and to account for non-isotropic heat radiation (Lebofsky et al. 1989). The STM is strongly connected to the IRAS minor planet catalogue (Tedesco et al. 2002), with more than 2000 diameter and albedo values derived from thermal observations. Morrison (1976) and Cruikshank \& Jones (1977) applied this technique for the first time to near-Earth asteroids (NEA). However, very early on Lebofsky et al. (1979) and Veeder et al. (1989) encountered problems when applying the STM to NEAs. They found in several cases albedos which were too high to be consistent with previous taxonomic classifications.

In recent years, Harris (1998) and Delbó \& Harris (2002) focused on the improvement of the radiometric technique for NEAs, resulting in the NEA thermal model (NEATM). The NEATM also incorporates a beaming parameter $\eta$, but now it is used as a variable which has to be adjusted to produce a fit to the spectral data. Harris (1998), Harris et al. (1998) and Delbó et al. (2003) applied the NEATM successfully to thermal $N$ and $Q$-band observations of a large number of NEAs. Harris \& Davies (1999) presented spectrophotometric observations of 3 NEAs and discussed the implications for their physical characterisation.

A thermophysical model (TPM) was developed by Lagerros (1996, 1997, 1998). A number of physical processes were introduced in this model. Instead of the empirical correction parameters, like the phase angle correction in the STM or the beaming parameter in the STM and NEATM, the TPM takes the true illumination and observing geometry into account and calculates the heat conduction into the surface. The non-isotropic emission, caused by the distribution of surface slopes, shadows, multiple scattering and mutual heating, is described by a 2-parameter beaming model with $f$, the fraction of the surface covered by craters, and $\rho$, the rms of the surface slopes. The TPM, as well as the STM, were mainly used for main-belt asteroids. It allows detailed thermophysical studies of individual asteroids (e.g., Müller \& Lagerros 1998) and predicts for well-known asteroids the thermal flux with high accuracy (Müller \& Lagerros 2002). Müller (2002) investigated the capabilities and limitations of the TPM in the context of NEA thermal observations.

Here we present $N$-band photometric and spectroscopic observations (Sect. 2). The interpretation of our 2002 NY40 observations was for the first time done with a combined NEATM and TPM approach (Sect. 3). We discuss the results of our

\footnotetext{
${ }^{5}$ http://www.ing.iac.es/PR/press/ing32002.html
}

analysis in Sect. 4. Using the case of 2002 NY40 as an example, we also demonstrate the possibilities and limitations of applying thermal models to asteroids during close Earth flybys.

\section{Observations and data reduction}

The 2002 NY40 observations were taken with the TIMMI2 instrument (Käufl et al. 2003) at the ESO La Silla $3.6 \mathrm{~m}$ telescope.

For all observations we utilized a standard chopping and nodding technique to reduce the atmospheric and telescope background emission. Chop and nod throws were 10", respectively. For the imaging observations, a pixel scale of $0.2^{\prime \prime}$ was chosen, and typical on source integration times of about $1 \mathrm{~min}$ for each filter were used. Exposure times for the low-resolution spectrum (resolution $\sim 160$ ) were between 90 and $220 \mathrm{~s}$.

The weather conditions were never ideal and parts of the two nights were affected by clouds. Table 1 contains only the good quality measurements together with the observing geometries.

The photometric N1-band flux was calibrated against measurements of HD 187642 and HD 12929. Colour differences between stars and 2002 NY40 were negligible (about 1-3\%). For observation No. 4 in Table 1 we obtained through standard aperture photometry a calibrated $8.6 \mu \mathrm{m}$ flux density of $22.2 \pm 1.6 \mathrm{Jy}$.

TIMMI2 spectroscopic data were reduced and analysed using procedures described by Siebenmorgen et al. (2004), including an airmass dependent extinction correction according to Schütz \& Sterzik (2004). The absolute flux level of TIMMI2 spectroscopic measurements is generally better than 5\% (Kämpgen \& Siebenmorgen 2004). However, our data are not as reliable mainly due to the fast speed of 2002 NY40 in combination with small slit sizes. Therefore, we normalised the spectra from August 18th to the N1 flux density. The spectra from the first day were normalised to a model flux (see Sect. 3). The spectra are shown in Figs. 1 and 2. The spectral shape is reliable in the range between about 8 and $13 \mu \mathrm{m}$.

Table 1. Summary of TIMMI2 observations of asteroid 2002 NY40 on August 17th/18th, 2002. The observations on August 17th were taken between airmass 1.2 and 1.3, on August 18th between 1.9 and 2.0. $r$, $\Delta$ and $\alpha$ are given as seen from La Silla. The spectra from August 17th were taken with a $1.2^{\prime \prime}$ slit, on August 18th with a $3^{\prime \prime}$ slit.

\begin{tabular}{rclllll}
\hline \hline & & $\begin{array}{l}\text { Mid-time } \\
\text { No. }\end{array}$ & $\begin{array}{l}\text { Filter } \\
\text { (Day UT) }\end{array}$ & $\begin{array}{l}r \\
\text { band }\end{array}$ & $\begin{array}{l}\Delta \\
{[\mathrm{AU}]}\end{array}$ & $\begin{array}{l}\alpha \\
{[\mathrm{AU}]}\end{array}$ \\
\hline 1 & 17 & $04: 48$ & Ngrism & 1.025406 & 0.013973 & 21.98 \\
2 & 17 & $04: 57$ & Ngrism & 1.025330 & 0.013900 & 22.06 \\
3 & 17 & $05: 07$ & Ngrism & 1.025245 & 0.013820 & 22.16 \\
4 & 18 & $02: 02$ & N1 & 1.014650 & 0.004535 & 58.37 \\
5 & 18 & $02: 08$ & Ngrism & 1.014600 & 0.004504 & 58.88 \\
6 & 18 & $02: 13$ & Ngrism & 1.014557 & 0.004478 & 59.30 \\
7 & 18 & $02: 18$ & Ngrism & 1.014515 & 0.004452 & 59.74 \\
8 & 18 & $02: 23$ & Ngrism & 1.014473 & 0.004427 & 60.17 \\
\hline
\end{tabular}




\section{Thermal modelling}

We applied the NEATM to determine radiometric diameter and albedo values from our N1-band photometric flux. For the beaming value we used $\eta=1.5$, as recommended by Delbó et al. (2003) for phase angles larger than $45^{\circ}$. As asteroid intrinsic input parameters we took an absolute magnitude of $H=19.6 \pm 0.2 \mathrm{mag}$ and a slope parameter $G=0.15 \pm 0.20$, based on an estimated extrapolation from 21 lightcurve observations between end of July and August 16th, 2002. The H-estimates for August 17th are much more accurate, but unfortunately our thermal photometry was not reliable for that day. Our observations of August 18th took place about $1 \mathrm{~h}$ after the lightcurve minimum (J. Licandro, personal communication).

The constraints from the optical and thermal measurements can be graphically shown (e.g., Sekiguchi et al. 2003) with respect to the NEATM assumptions and formulae (e.g., Delbó \& Harris 2002). Our NEATM analysis resulted in an effective diameter of $0.27 \pm 0.01 \mathrm{~km}$ and an albedo value of $0.34+0.06 /-0.05$ (Fig. 3)

Based on these values, we determined a NEATM $8.6 \mu \mathrm{m}$ flux density of $5.9 \pm 0.8 \mathrm{Jy}$ for the August 17 th spectra, which we used for normalisation (see Fig. 1). This NEATM prediction is based on the Delbó et al. (2003) recommendation to take a value of $\eta=1.0$ for data taken at phase angles below $45^{\circ}$.

For both days we calculated spectral energy distributions for the mean epochs of the $N$-band spectra through the STM, the Fast Rotating Model (FRM; e.g., Lebofsky \& Spencer 1989 ) and the NEATM with $\eta=1.0$ and $\eta=1.5$. A nice summary of all formulae can be found in Delbó \& Harris (2002). The model predictions are shown in Figs. 1 and 2.

We also applied the TPM by Lagerros (1996, 1997, 1998) to our observations. We assumed a spherical asteroid with an

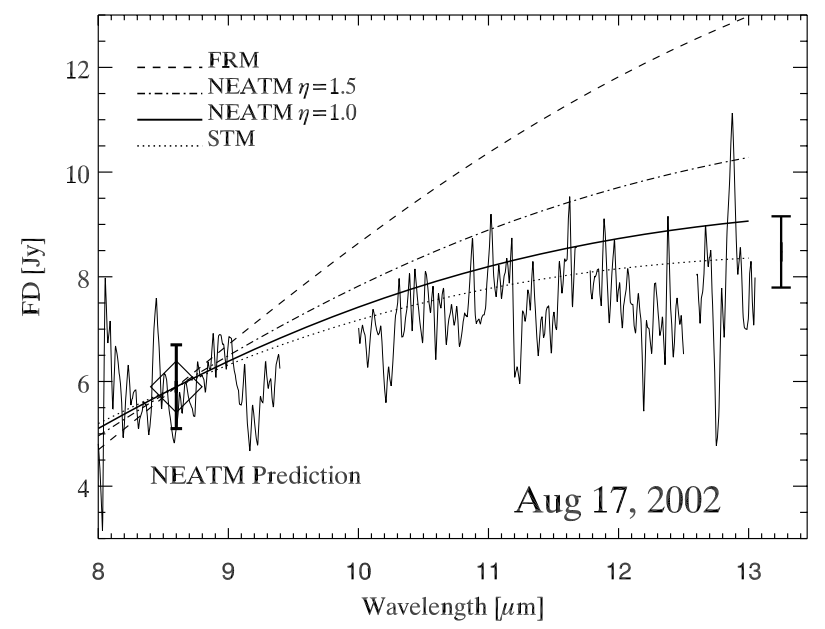

Fig. 1. The best $N$-band spectrum of 2002 NY40 from August 17th, 2002 (No. 3 in Table 1). It was spectroscopically calibrated against HD 133774 and against HD 187642 and then averaged. The $1 \sigma$-error bar represents the signal processing error together with the $1 \sigma$-average error (on the right side). The residual atmospheric features (ozone around $9.58 \pm 0.3 \mu \mathrm{m}$ and $\mathrm{CO}_{2}$ around 11.73 and $12.55 \mu \mathrm{m}$ ) were cut out. The spectra were normalised to a NEATM $(\eta=1.0)$ prediction at $8.6 \mu \mathrm{m}$ for the given observing epoch.

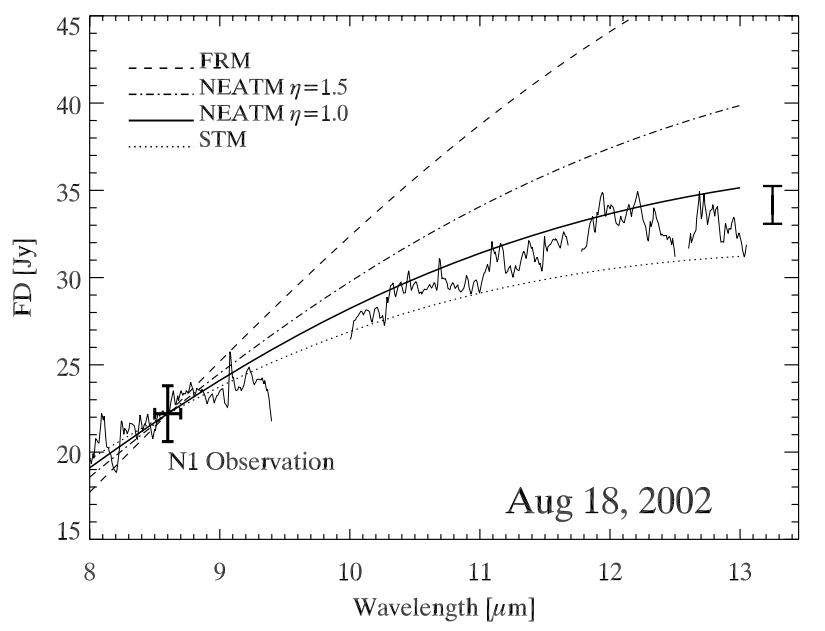

Fig. 2. The average of the two best $N$-band spectra of 2002 NY40 on August 18th, 2002 (Nos. 5 and 6 in Table 1), both calibrated against HD 187642. The error bar was calculated in the same way as for Fig. 1. The residual atmospheric features were cut out and the spectra were normalised to the N1-band photometric flux.

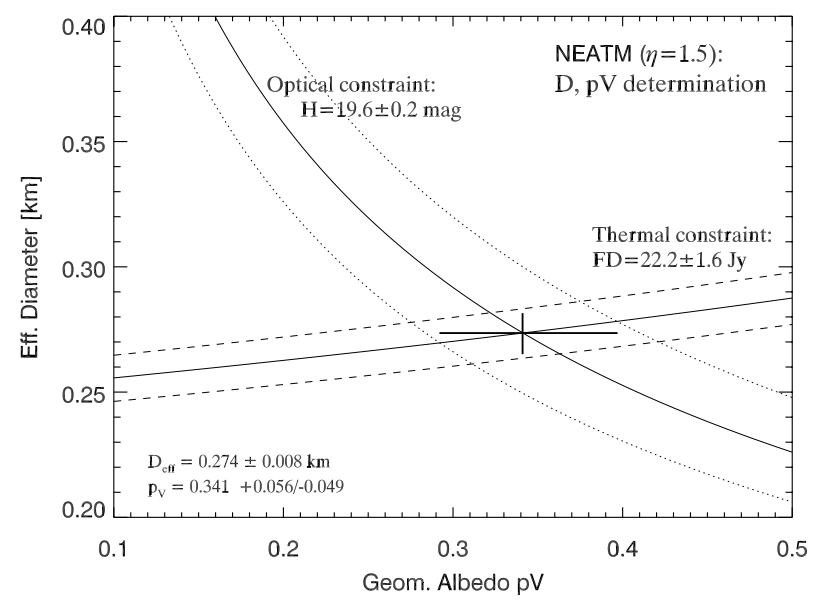

Fig. 3. NEATM analysis of the N1-band photometric flux. The $\mathrm{H}$-estimate is relatively uncertain and propagates mainly into the uncertainty of $p_{V}$, while the $D_{\text {eff }}$ uncertainty is dominated by the thermal flux which is relatively accurate. The $D_{\text {eff }}$ and $p_{V}$ results are connected to the assumption that the model does not introduce a systematic error.

equator-on viewing angle under the given phase angle and a prograde rotation with $P_{\text {sid }}=19.98 \mathrm{~h}$ (Pravec et al. 2004). Müller et al. (1999) derived $f=0.6$ and $\rho=0.7$ for large regolith-covered main-belt asteroids. In addition, wee included a relatively smooth surface with a low crater coverage $(f=0.4$, $\rho=0.4$ ) and a very rough surface with $100 \%$ crater coverage $(f=1.0, \rho=1.0)$, similar to the studies by Dotto et al. (2000) and Müller (2002). For the emissivity, we used a constant, wavelength-independent value of 0.9 .

The thermal behaviour of asteroids is strongly coupled to the thermal inertia $\Gamma$, in combination with the orientation of the body in space, its rotation period and the sense of rotation. A high $\Gamma$ combined with a rapid rotation (sub-solar and subobserver points on the equator) would lead to a surface temperature distribution described by the FRM. A non-rotating, low $\Gamma$ object would correspond to the STM temperature distribution. Here, we varied $\Gamma$ between 10 (regolith covered surface 
Table 2. The radiometric diameter and albedo values for the different models based on $\mathrm{N}$-band photometry. The values are discussed in Sect. 4.

\begin{tabular}{llll}
\hline \hline Model & Diameter $[\mathrm{km}]$ & Albedo & Remarks \\
\hline STM & $0.20 \pm 0.02$ & $0.64_{-0.08}^{+0.09}$ & $\eta=0.756$ \\
FRM & $0.30 \pm 0.02$ & $0.29_{-0.04}^{+0.05}$ & \\
NEATM & $\mathbf{0 . 2 7} \pm \mathbf{0 . 0 1}$ & $\mathbf{0 . 3 4}_{-0.05}^{+0.06}$ & $\eta=1.5$ \\
NEATM & $0.22 \pm 0.01$ & $0.52_{-0.07}^{+0.08}$ & $\eta=1.0$ \\
TPM & $\mathbf{0 . 2 8} \pm \mathbf{0 . 0 3}$ & $\mathbf{0 . 3 5} \pm \mathbf{0 . 0 2}$ & $\Gamma=1000$ \\
TPM & $0.23 \pm 0.03$ & $0.51 \pm 0.03$ & $\Gamma=100$ \\
\hline
\end{tabular}

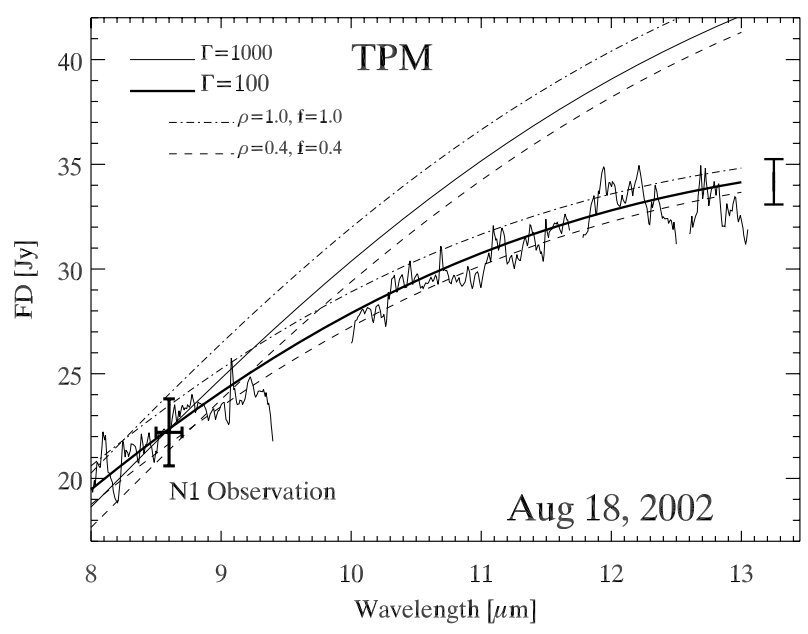

Fig. 4. $N$-band spectrum of 2002 NY40 from August 18th, 2002, with the error bar as in Fig. 2. TPM predictions with $\Gamma=1000$ (upper solid line) and $\Gamma=100 \mathrm{~J} \mathrm{~m}^{-2} \mathrm{~s}^{-0.5} \mathrm{~K}^{-1}$ (lower solid line) are shown. The dashed and dashed-dotted lines indicate the influence of surface roughness expressed through $\rho$ and $f$ for both values of $\Gamma$, respectively. Default values are $f=0.6$ and $\rho=0.7$.

at about $100 \mathrm{~K}$ ) and $2500 \mathrm{~J} \mathrm{~m}^{-2} \mathrm{~s}^{-0.5} \mathrm{~K}^{-1}$ (solid rock, Jakosky 1986).

In order to obtain a similar diameter and albedo value as produced by the NEATM calculations, we had to use a high $\Gamma$ value of about $1000 \mathrm{~J} \mathrm{~m}^{-2} \mathrm{~s}^{-0.5} \mathrm{~K}^{-1}$. With these values we obtained a TPM diameter of $0.28 \pm 0.03 \mathrm{~km}$ and an albedo of $0.35 \pm 0.02$. The errors include the uncertainties in the measured flux density, the given $\mathrm{H}-\mathrm{G}$ variations, the above specified range of $(\rho, f)$-values and a $20 \%$ variation in $\Gamma$.

In Table 2, we summarise the radiometric diameter and albedo values for different models applied to observation No. 4 (Table 1). As a combined NEATM/TPM result we took $D_{\text {eff }}=$ $0.28 \pm 0.03$ and $p_{V}=0.34 \pm 0.06$.

Figure 4 shows the TPM solution for the August 18th spectra with $\Gamma=1000 \mathrm{~J} \mathrm{~m}^{-2} \mathrm{~s}^{-0.5} \mathrm{~K}^{-1}$ and $\Gamma=100 \mathrm{~J} \mathrm{~m}^{-2} \mathrm{~s}^{-0.5} \mathrm{~K}^{-1}$. For both models the influences of a relatively smooth and a very rough surface are shown as dashed and dashed-dotted lines. The corresponding figure for the August 17th spectrum is very similar. The $\Gamma=100$ solution matches nicely, the $\Gamma=1000$ solution is steeper and at long wavelengths significantly above the observed spectrum.

\section{Discussion}

Our analysis was guided by the radar size estimate of about $250 \times 420 \mathrm{~m}($ E. Howell, personal communication) and the radiometric results (Rivkin et al. 2003) of a 290-420 m diameter and an albedo preference of about 0.25 . All model calculations (see Table 2) produced diameters below or at the lower end of these numbers, while the albedo was in all cases 0.29 or larger.

The STM of Lebofsky et al. (1986) has a phase angle correction of $\beta_{E}=0.01 \mathrm{mag} \mathrm{deg}^{-1}$, which is considered to be a good approximation for phase angles $\leq 30^{\circ}$ (Morrison 1977). For our photometry at $59^{\circ}$ it is therefore not a good option. The resulting diameter and albedo values are extreme and far away from the radar size or the albedo of a Q-class asteroid. The STM fit to the spectral slope is astoundingly good, only for the August 18th data the STM prediction falls slightly below the NEATM prediction.

The FRM led to an acceptable agreement with the radar data. But the FRM did not match the spectral shape of the two spectroscopic data sets (Figs. 1 and 2).

The NEATM $(\eta=1.5)$ and the TPM $(\Gamma=1000)$ produced solutions which are comparable to the radar size, but again they cannot match the observed SEDs. On the other hand, a NEATM with $\eta=1.0$ or a TPM with $\Gamma=100$ match the spectra, but produce an unrealistically small diameter of about 220-230 m and a very high albedo above 0.50 . We do not have a convincing explanation for this, but with only one reliable photometric flux it is difficult to find a solution, unless 2002 NY40 turns out to be a small, very high albedo object. It might also be that strong deviations from a spherical shape seen under the large phase angle of $58.37^{\circ}$ cause thermal effects which are difficult to model. On the other hand, one set of model parameters fits all spectra, although they were taken at very different phase angles. This is true for the NEATM and the TPM.

The NEATM $8.6 \mu \mathrm{m}$ prediction for the spectra of the first day are $5.9 \mathrm{Jy}(\eta=1.0)$ and $3.6 \mathrm{Jy}(\eta=1.5)$. The corresponding TPM calculations (taking also the NEATM diameter and albedo) give $6.8 \mathrm{Jy}(\Gamma=100)$ and $4.1 \mathrm{Jy}(\Gamma=1000)$. A reliable photometry for this day would therefore indicate which of the models has the better simulation of the thermal phase angle effects.

We cannot confirm a phase angle trend on the beaming parameter as proposed by Delbó et al. (2003). A NEATM solution with $\eta=1.0$ fits the spectra of both days at phase angles of about $22^{\circ}$ and $59^{\circ}$.

The $\Gamma$-value is very crucial for our TPM analysis. A value of $\Gamma=15 \mathrm{~J} \mathrm{~m}^{-2} \mathrm{~s}^{-0.5} \mathrm{~K}^{-1}$, as it was used for regolith-covered main-belt asteroids (Müller et al. 1999), leads to a very small diameter and an albedo of about 0.6. Taking the lunar soil value of $\Gamma=39 \mathrm{~J} \mathrm{~m}^{-2} \mathrm{~s}^{-0.5} \mathrm{~K}^{-1}$ (Keihm 1984) does not improve the situation much. Only large $\Gamma$-values in the order of $1000 \mathrm{~J} \mathrm{~m}^{-2} \mathrm{~s}^{-0.5} \mathrm{~K}^{-1}$ give reasonable results. However, such a large value can only be the case if 2002 NY40 has a surface with no or little regolith. A dust layer on the surface would always produce much lower thermal inertias.

Through a TPM analysis it is also possible to investigate the sense of rotation (e.g., Müller 2002). We assumed a prograde rotator meaning that the terminator is coming from the cold 
night side of the asteroid on August 18th. Taking a retrograde rotator, with the terminator coming from the warm day side, would require a $\Gamma$-value of about $3000 \mathrm{~J} \mathrm{~m}^{-2} \mathrm{~s}^{-0.5} \mathrm{~K}^{-1}$ to obtain a diameter similar to the radar diameter. Such value would be greater than the value for solid rock (Jakosky 1986) and therefore not very likely. Again, with only one reliable flux it is difficult to obtain a final conclusion, but the TPM analysis points to a prograde rotator with a cold terminator after opposition. This result will also be useful in case of a possible ambiguous solution of its pole orientation in the future.

The TPM curves in Fig. 4 demonstrate that the beaming model, expressed in $\rho$ and $f$, has no big influence on the 8 to $13 \mu \mathrm{m}$ predictions. Distinguishing between a very rough, cratered surface with pronounced thermal beaming effect and a relatively smooth surface with no enhanced radiation at small phase angles was therefore not possible for our data set. But the beaming does influence the flux differences between two phase angles (see also Müller 2002). Thermal observations with a good coverage in phase angle in combination with a few additional spectra would give the possibility to determine $\Gamma$ and indicate if the asteroid has a rough, cratered and therefore old surface or a relatively smooth, young surface.

Rivkin et al. (2003) identified olivine and pyroxene in the near-IR spectra. The most diagnostic feature in the $N$-band is the Christiansen peak. It is associated with the principal molecular vibration band where the refractive index changes rapidly. For silicates it appears generally as peak between 7.5 and $9.5 \mu \mathrm{m}$ (Dotto et al. 2002). We did not see any prominent increase in emittance in this range. The feature might have been just outside the reliable wavelength range or the quality of the spectra was not sufficient to identify silicate features.

\section{Conclusion}

Guided by the radar size, we found that both the NEATM with $\eta=1.5$ and the TPM with $\Gamma=1000 \pm 200 \mathrm{~J} \mathrm{~m}^{-2} \mathrm{~s}^{-0.5} \mathrm{~K}^{-1}$ produce consistent effective diameter values of $0.28 \pm 0.03 \mathrm{~km}$ for the time of the $N$-band photometric observation. The corresponding geometric albedo is $0.34 \pm 0.06$.

Neither of the two model solutions give a satisfying match to the spectra obtained in two observing sessions, separated by slightly more than one rotation period. A match of the spectra required either a NEATM with $\eta=1.0$ or a TPM with a thermal inertia in the order of $100 \mathrm{~J} \mathrm{~m}^{-2} \mathrm{~s}^{-0.5} \mathrm{~K}^{-1}$. No phase angle dependence of the NEATM $\eta$-parameter can be seen in our data.

The TPM analysis excluded a dust-covered surface and pointed towards a bare rock surface or a very coarse regolith. We obtained reasonable values for the thermal inertia, the diameter and albedo, but only assuming a prograde rotation. This means that we saw a cold terminator during the observations which took place when 2002 NY40 was just after opposition, but before its closest Earth-approach.

The example of 2002 NY40 shows the different possibilities to analyse thermal observations of NEAs through the NEATM and the TPM. A carefully planned observing campaign under photometric conditions and covering a wide range in solar phase angle (without large changes in the aspect angles) are ideal for this kind of investigations. High quality photometric fluxes are the key to distinguish between different model approaches, but a good coverage in visual lightcurves is also required to determine $H$-/ $G$-values and the orientation of the object at the time of the thermal observations. Additional calibrated $N$-band spectra allow for estimates of the thermal inertia to prove the existence of a dust regolith and the sense of rotation. The August 17th/18th 2002 fly-by of 2002 NY40 was in principle an ideal case for such a study. Only better atmospheric conditions for mid-IR observations and a dedicated campaign in the optical would have provided more accurate results. Close fly-bys at distances below $0.01 \mathrm{AU}$ are quite frequent (the MPC lists currently 14 such event for the period January to May 2004), but most of these events are only known on short notice or after the fly-by. In fact, there is not even one such event listed the period June 2004 to December 2009. The next close Earth approach of 2002 NY40 will be on July 26th, 2005 , but at a relatively large distance of $0.43 \mathrm{AU}$.

Acknowledgements. We would like to thank A. W. Harris (DLR) and M. Delbó for their support in reproducing their NEATM model code.

\section{References}

Bowell, E., Hapke, B., Domingue, D., et al. 1989, in Asteroids II, ed. R. P. Binzel, T. Gehrels, \& M. S. Matthews (Arizona University Press), 524

Clark, B. E., Hapke, B., Pieters, C., \& Britt, D. 2002, in Asteroids III, ed. W. F. Bottke Jr., A. Cellino, P. Paolicchi, \& R. P. Binzel (Tucson: University of Arizona Press), 585

Cruikshank, D. P., \& Jones, T. J. 1977, Icarus, 31, 427

Delbó, M., \& Harris, A. W. 2002, Meteoritics \& Planetary Science, 37,1929

Delbó, M., Harris, A. W., Binzel, R. P., Pravec, P., \& Davies, J. K. 2003, Icarus, 166, 116

Dotto, E., Müller, T. G., Barucci, M. A., et al. 2000, A\&A, 358, 1133

Dotto, E., Barucci, M. A., Müller, T. G., et al. 2002, A\&A, 393, 1065

Harris, A. W. 1998, Icarus, 131, 291

Harris, A. W., Davies, J. K., \& Green, S. F. 1998, Icarus, 135, 441

Harris, A. W., \& Davies, J. K. 1999, Icarus, 142, 464

Howell, E. S., Rivkin, A. S., Nolan, M. C., et al. 2003, Abstract book of the IAU Conference, Sydney, July 2003, JD 19, Abstract No. 1872,254

Jakosky, B. M. 1986, Icarus, 66, 117

Kämgen, K., \& Siebenmorgen, R. 2004, in High Resolution Infrared Spectroscopy in Astronomy, ed. H. U. Käufl, R. Siebenmorgen, \& A. Moorwood (Springer-Verlag), in press

Käufl, H. U., Sterzik, M., \& Siebenmorgen, R. 2003, SPIE, 4841, 117

Keihm, S. J. 1984, Icarus, 60, 568

Lagerros, J. S. V. 1996, A\&A, 310, 1011

Lagerros, J. S. V. 1997, A\&A, 325, 1226

Lagerros, J. S. V. 1998, A\&A, 332, 1123

Lebofsky, L. A., Lebofsky, M. J., \& Rieke, G. H. 1979, AJ, 84, 885

Lebofsky, L. A., Sykes, M. V., Tedesco, E. F., et al. 1986, Icarus, 68, 239

Lebofsky, L. A., \& Spencer, J. R. 1989, in Asteroids II, ed. R. P. Binzel, T. Gehrels, \& M. S. Matthews (Arizona University Press), 128

Morrison, D. 1976, Icarus, 28, 125

Morrison, D. 1977, ApJ, 214, 667

Morrison, D., \& Lebofsky, L. 1979, 184 in Asteroid, ed. T. Gehrels (Tucson: University of Arizona Press)

Müller, T. G., \& Lagerros, J. S. V. 1998, A\&A, 338, 340 
Müller, T. G., Lagerros, J. S. V., Burgdorf, M., et al. 1999, ESA SP427, in The Universe as Seen by ISO, ed. P. Cox, \& M. F. Kessler, 141

Müller, T. G., \& Lagerros, J. S. V. 2002, A\&A, 381, 324

Müller, T. G. 2002, M\&PS, 37, 1919

Pravec, P., Harris, A. W., Scheirich, P., et al. 2004, Icarus, 2004, submitted

Rivkin, A. S., Howell, E. S., Bus, S. J., et al. 2003, 34th Annual Lunar and Planetary Science Conference March 2003, Abstract No. 1722, http://www. mit. edu/ asrivkin/lpsc03B.pdf
Schütz, O., \& Sterzik, M. 2004, in High Resolution Infrared Spectroscopy in Astronomy, ed. H. U. Käufl, R. Siebenmorgen, \& A. Moorwood (Springer-Verlag), in press [arXiv: astro-ph/0404200]

Sekiguchi, T., Abe, M., Böhnhard, H., et al. 2003, A\&A, 397, 325

Siebenmorgen, R., Krügel, E., \& Spoon, H. W. W. 2004, A\&A, 414, 123

Tedesco, E. F., Noah, P. V., Noah, M., \& Price, S. D. 2002, AJ, 123, 1056

Veeder, G. J., Hanner, M. S., Matson, D. L., et al. 1989, AJ, 97, 1211 